\title{
Author Correction: The mitochondrial protein Opa1 promotes adipocyte browning that is dependent on urea cycle metabolites
}

Camilla Bean, Matteo Audano, Tatiana Varanita, Francesca Favaretto, Marta Medaglia, Marco Gerdol(10, Lena Pernas, Fabio Stasi, Marta Giacomello D, Stèphanie Herkenne, Maheswary Muniandy @ D Sini Heinonen, Emma Cazaly,

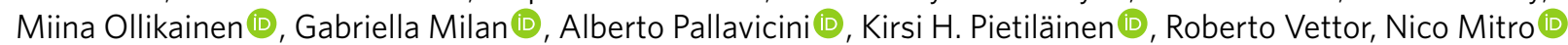
and Luca Scorrano

Correction to: Nature Metabolism https://doi.org/10.1038/s42255-021-00497-2, published online 6 December 2021.

In the version of this article initially published, Maheswary Muniandy's first name was misspelled in the author list as Maheswany. The name has been corrected in the HTML and PDF versions of the article.

Published online: 11 February 2022

https://doi.org/10.1038/s42255-022-00548-2

(c) The Author(s), under exclusive licence to Springer Nature Limited 2022 